\title{
BIRDQUESTVR:
}

\section{A CROSS-PLATFORM ASYMMETRIC COMMUNICATION GAME}

\author{
by \\ Michael Smilovitch \\ BASc, McGill University, 2016 \\ A Major Research Paper \\ presented to Ryerson University \\ in partial fulfillment of the \\ requirements for the degree of \\ Master of Digital Media \\ in the program of \\ Digital Media \\ Toronto, Ontario, Canada, 2019 \\ (C) Michael Smilovitch, 2019
}




\begin{abstract}
Author's Declaration
I hereby declare that I am the sole author of this MRP. This is a true copy of the MRP, including any required final revisions.

I authorize Ryerson University to lend this MRP to other institutions or individuals for the purpose of scholarly research.

I further authorize Ryerson University to reproduce this MRP by photocopying or by other means, in total or in part, at the request of other institutions or individuals for the purpose of scholarly research.
\end{abstract}

I understand that my MRP may be made electronically available to the public. 


\title{
BIRDQUESTVR: A CROSS-PLATFORM ASYMMETRIC COMMUNICATION GAME
}

Michael Smilovitch

Master of Digital Media

Ryerson University, 2019

\begin{abstract}
BirdQuestVR is a cross-platform asymmetric communication game between one player in Virtual Reality and another on a mobile device. The game explores asymmetric co-operative gaming in a shared physical space, taking the physical surroundings of the VR user into account in its design. Asymmetric games feature different rules, abilities, or objectives for different players, generating unique and nuanced game experiences. Multiplayer asymmetric games in particular have been shown to increase teamwork and a collaborative mindset even after a play session has ended. Asymmetric design is commonplace in both digital and analog games but has yet to see widespread adoption in the emerging Virtual Reality (VR) gaming space. BirdQuestVR seeks to leverage the affordances of current consumer-grade VR headsets to build asymmetric gameplay around communication, embodied performance, and physical humour.
\end{abstract}

Keywords: Asymmetric, Virtual Reality, Cross-Platform, Social Play, Avatar Embodiment 


\section{Acknowledgements}

This work was carried out as a Ryerson Master of Digital Media Major Research Paper/Prototype. I would like to acknowledge the ongoing support of the Ryerson Transmedia Zone on the project.

This project would not be possible without the feedback and contributions of Andreas Arteaga, Ebyan Bihi, Samantha Fernandez, Carlos Ferro, Stefan Gambert, Teodor Herman,

Nathaniel Lam, Kathryn Mackenzie, Kadrah Mensah, and Wayne Shipley, along with everyone who offered their time for user testing and feedback.

The playable prototype was built using the Unity game engine and uses Normcore, a plugin by Normal VR, for networking. 


\section{Table of Contents}

Author's Declaration $\quad$ ii

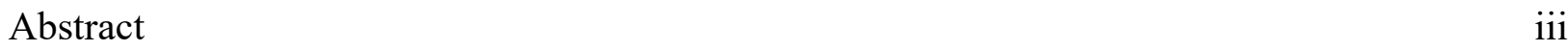

Acknowledgements

Table of Contents $\quad$ V

List of Figures $\quad$ vi

Introduction $\quad 1$

Social Gaming in Virtual Reality 2

$\begin{array}{ll}\text { The Physicality of VR } & 3\end{array}$

Related Works

$\begin{array}{lr}\text { Hardware } & 6\end{array}$

$\begin{array}{ll}\text { Game Design } & 7\end{array}$

$\begin{array}{ll}\text { Future Work } & 10\end{array}$

$\begin{array}{ll}\text { References } & 11\end{array}$ 


\section{List of Figures}

Figure 1. The tablet player's view

Figure 2. The VR player's view 


\section{Introduction}

In many games, competition exists between players bound by identical rules, with matched or balanced in-game abilities. These games are symmetric [8]. Asymmetric gameplay occurs in a game environment where different players have different rules, abilities, or objectives $[2,3,4,8]$. A classic example of asymmetric gameplay is in the 1970 code-breaking game "Mastermind", in which where one player must select a hidden sequence of colours, and the other must deduce the sequence within a limited number of turns [11]. Asymmetric gameplay is common across digital games [8] and has been shown to foster teamwork [4], critical thinking [3], and negotiation skills [2] more effectively than symmetric gamely.

Asymmetric gameplay is common within collaborative games as well as competitive ones. In many online digital games, asymmetry can exist within teams, in the form of different "heroes", or "classes" that may feature different playstyles. It has become the norm in online games for players on a team to have different complementary abilities, as this setup inherently promotes teamwork [4]. For example, many popular online games such as Overwatch and League of Legends feature "support" characters, whose roles are to primarily to heal or protect the team's main damage-dealer. It's much easier to have a distinct player for healing, and a distinct player for damage-dealing, so that complementary roles can be established within a team [18].

Asymmetric game design can also allow for players of differing skill levels to play together, such as the option for one player to use a touch interface to make small contributions to gameplay in "New Super Mario Bros U" [15]. Asymmetric design can promote inclusivity in games, where players can find roles best suited to their unique preferences and capabilities [16]. 


\section{Social Gaming in Virtual Reality}

The recent resurgence of Virtual Reality (VR) gaming has provided a host of new opportunities for explorative and unique game design. While there are plenty of interesting single-player games available, a recent report by Greenlight Insights suggests that $77 \%$ of VR users want more social engagement [29]. While there are plenty of multiplayer VR games, most are online, meaning players aren't in the same shared physical space. Further, these games require a VR headset per person.

Headsets are rapidly becoming more powerful and portable, such as the newly released wireless Oculus Quest [22]. The added portability of these devices, and future devices, will generate more situations in which VR is being shared socially, as it can now be carried around much more easily than before these wireless solutions. At the same time, VR arcades are popping up worldwide, creating more opportunities for people to experience VR in a social setting [21].

Whether at home or at the arcade, the social dynamics in the physical environment is one which is neglected in existing literature and in commercial designs. We suggest a design opportunity for people outside of VR (henceforth referred to as spectators), bearing in mind the physical and social contexts in which people will be playing VR. 


\section{The Physicality of VR}

In his GDC talk, “The Future of Storytelling: How Medium Shapes Story”, game designer Jesse Schell explains that we should not design for consoles, but instead for "venues" [20]. He argues that while hardware may change, the places where we engage with games will stay the same.

Most VR experiences design strictly for what happens within the headset, leaving out many important components of experience. While some people might be playing alone in private, many will be experiencing VR with friends at home or at VR arcades. In these scenarios, there are people waiting their turn for the headset, or simply watching and socializing. These spectators, a key component of the user experience of the moment, and what we will refer to as a VR game's physical context, are typically not acknowledged in game design. The design-space of the game is, often, strictly what happens within the headset.

The widely used Experience Design Model, proposed by Conifer Research, outlines five stages to a user experience: "Entice, Enter, Engage, Exit, and Extend" [19]. Each "E" presents an opportunity to enhance the user experience above and beyond the core game itself (the "Engage" moment in this model). The user experience is more than just the game itself, but rather includes the context surrounding the game: what the environment is like before we don the headset, and after we take it off; our preconceptions formed while watching people in line ahead of us play through an unfamiliar experience; and the dynamics of our social interactions with

friends and strangers before and after the in-headset game. Each element can affect how we feel about the game, along with our experience of the game itself. 
In order to design for physical context, we looked at some of the basic affordances of VR. It is undeniable that people in VR look rather silly. Not only are they wearing bulky hardware, they are often unable to hear the outside world, and lose all sense of orientation. This is funny to watch. With BirdQuestVR, we set out to design a cross-platform VR game that would allow multiple people to play a game using a single VR headset. Our rationale for the single headset was that this would create more opportunities for social play when there is only a single headset available, which we assume will often be the case as more people have their first VR experiences. Our design philosophy was to embrace the physicality of VR, and the inherent silliness of having one person in a VR headset with others outside, watching.

Because VR relies so heavily on immersion, that is, the feeling of truly being present inside a virtual environment, designers may feel that if a player were to acknowledge people outside the game, this would break immersion due to the acknowledging of a parallel, non-game environment [6]. On the contrary, social interaction in digital, non-VR multiplayer games has been shown to increase immersion, because it introduces an element of role-play and allows players to connect more deeply with their respective in-game roles [5].

With BirdQuestVR, we treat cross-platform asymmetric gameplay within VR as an opportunity for social interaction between VR players and spectators that doesn't break immersion, but instead increases it. 


\section{Related Works}

There are several existing VR games that support asymmetric multiplayer. Some allow non-VR players to engage with gameplay via mobile phones, such as Statik and Final Soccer VR $[25,26]$. Others, like GORN, let non-VR players join with a controller and fight against the VR player [27].

The most popular asymmetric multiplayer VR game is undoubtedly "Keep Talking and Nobody Explodes", a game in which one player in VR must defuse a bomb, while someone outside of VR has access to the instruction manual [24]. The primary game mechanic becomes conversation, and neither player has all the tools to succeed on their own - a key to good collaborative design [18]. "Keep Talking and Nobody Explodes" was one of our greatest inspirations for how to approach asymmetric VR game design. 


\section{Hardware}

With BirdQuestVR, we set out to create an asymmetric VR party game. A key component of the design philosophy was bearing in mind the context in which the game might be played, namely, keeping in mind those who are outside of VR, as much the player who is in within VR.

We didn't want to only use conversation, but also experiment with the use of gestural communication. The newly released Oculus Quest is the first ever untethered headset with 6 Degrees of Freedom (6DOF) tracking. 6DOF means that VR players can not only rotate to examine their environment but can also move up and down and side to side, walking through the virtual world. 6DOF headsets typically also feature controllers, allowing for VR users to feel as though they have hands in the world - a key to establishing immersion [28].

The Oculus Quest headset is the first ever VR headset to feature 6DOF tracking without external sensors, making it the first truly portable headset of its kind [22]. We chose to target the Oculus Quest device for the VR portion of BirdQuestVR, due to the unique affordances provided by $6 \mathrm{DOF}$ tracking - virtual hands and the ability to gesture. 


\section{Game Design}

Many of the social benefits associated with asymmetric collaborative gaming rely on interdependence $[5,16]$, meaning no one player has all the information or tools needed to advance. We chose therefore to make communication crucial to success in BirdQuestVR. The current version of BirdQuestVR is designed for two players: one in VR on an Oculus Quest, and one on a mobile device (we are using a Samsung Galaxy Tab S2 for our tests).

In BirdQuestVR, the VR player embodies "Captain Nugget P. Rotisserie", a chicken in space on an important mission. The tablet player plays as mission control, or "H.E.N", remotely helping Captain Nugget complete the mission.

Each player has access to several ship systems. The VR player can turn engines on or off by physically reaching out and pressing buttons, while the tablet player can control a series of "boosters" and "roosters" by tapping buttons on their mobile device.

To score points, both players must follow instructions in order to power up the proper systems. To ensure interdependence, players receive the instructions for the other player. For example, one communication might be "Set boosters to ON/OFF/ON". The VR player will have this information, but the tablet player is the one with control of the boosters, making communication a crucial key to progress.

The mobile player's UI is centred around a live video feed of the VR player (or "Chicken Feed", as we call it). This is to emphasize the game's focus on communication through performance. The VR player appears on the mobile device as an avatar - a floating head and hands. 
In early tests, we didn't allow users to talk. This led to a lot of confusion regarding roles in gameplay. In the current version, players can both talk for the first half of the game (one minute), before we begin to alter communication. As the game continues, the VR player must begin to express themselves using gestures and physical movements from within VR, acting as the VR character rather than as themselves. We justify this disruption in-game by claiming that the "translation module" has stopped working, and that the VR player can no longer speak to the mobile player. Instead of the VR player's voice, the tablet player can only hear clucking for this second half of gameplay.

Suddenly, gestural communication becomes the emphasis of the game. For players to progress, they must develop a system of communicating using only the VR avatar on the tablet. The added bonus of this is the humour in how the VR player appears to spectators. This spurs creativity and emergent behavior, as there is no "correct" way to communicate via gestures: it's up to the players to develop their own system.

To amplify our focus on performance and entertainment for those outside of VR, we experimented with altering the appearance of the Oculus Quest headset. Using simple materials, we created a version of our VR character's head to affix to the headset. We also provide mission control, or H.E.N., with a lab-coat in order for them to feel more scientific.

Having the VR player physically look like a space-chicken in real-life, while embodying one in VR, adds to the visual spectacle of the entire experience, both enticing and amusing others who may be in the same physical space. 


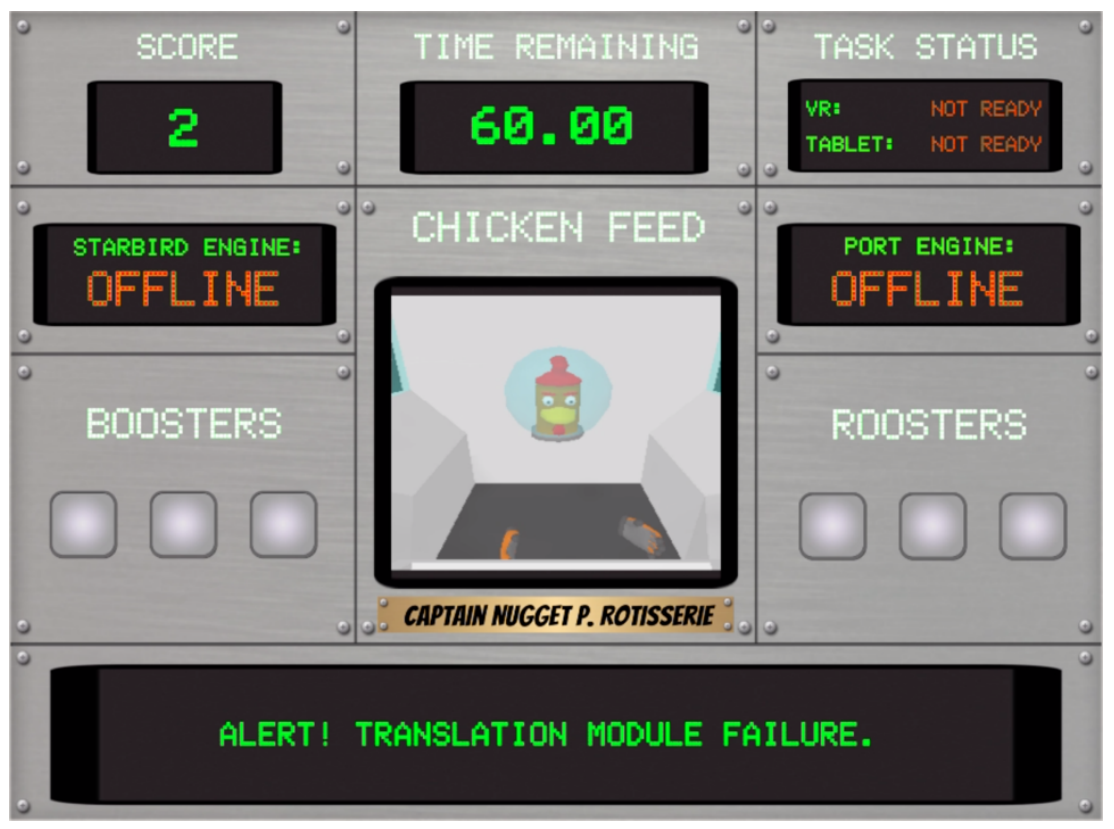

Figure 1 - The tablet player's view

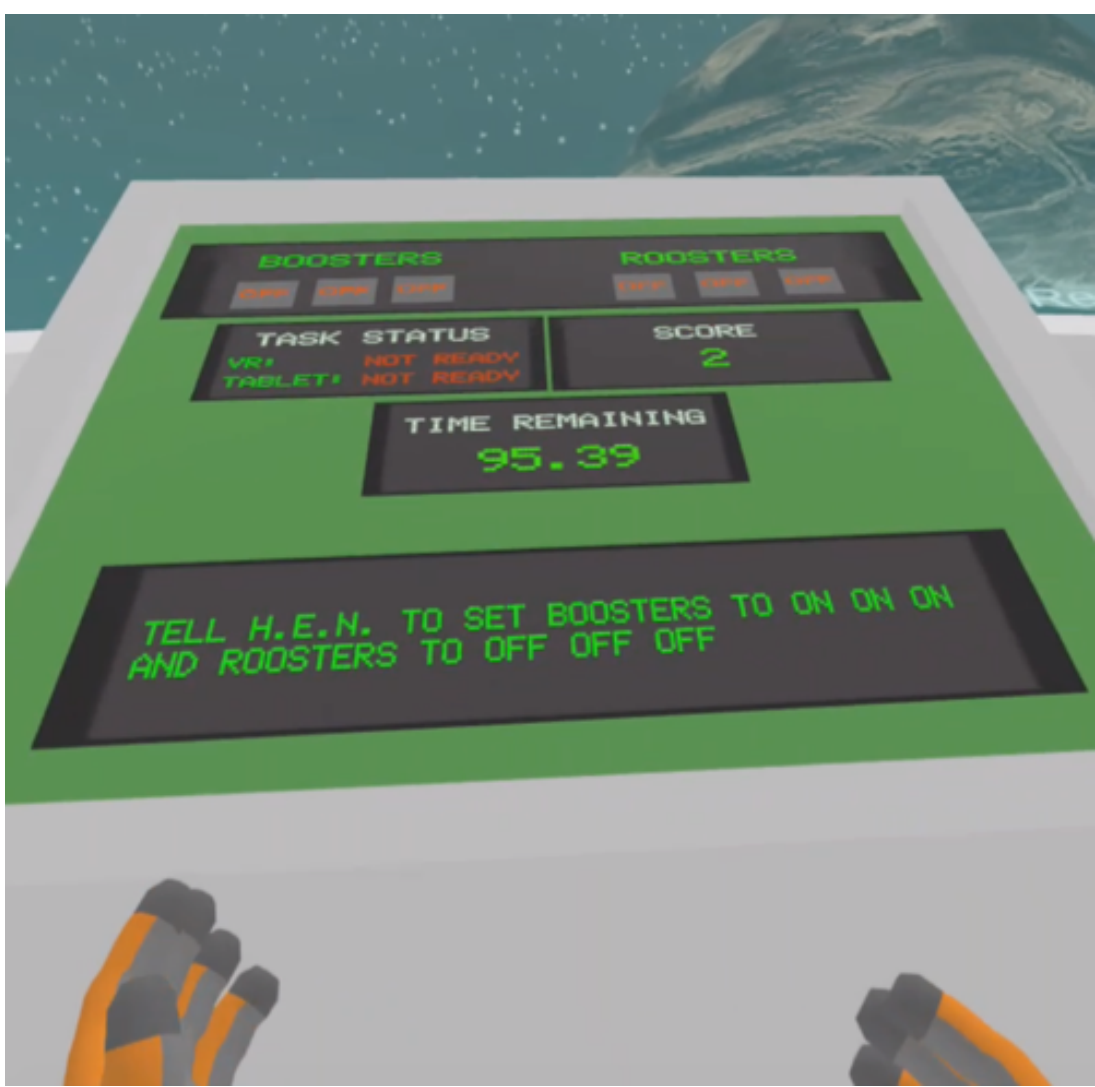

Figure 2 - The VR player's view 


\section{Future Work}

We have a range of design elements we would like to add to the game over time in order to further develop our approach to gaming. For example, we would like to develop the ability for players to use more precise gestures. The Oculus Quest controllers support a few hand configurations, such as pointing, or making a "thumbs up" which would allow for more creative communication options and emergent gameplay. For even higher-quality finger tracking, we could try implementing a version that leverages Leap Motion for controller-less finger tracking. Finally, support for headsets such as the Vive and Oculus Rift as well as the inclusion of more puzzles and scenarios should add to the game's overall appeal. 


\section{References}

1. Tynan Sylvester. 2013. Designing games. O'Reilly Media, Sebastopol, CA.

2. Nicolas Becu, Nathalie Frascaria-Lacoste, and Julie Latune. (2015). Experiential learning based on the NewDistrict asymmetric simulation game : results of a dozen gameplay sessions. Hybrid Simulation Gaming in the Networked Society, 2015.

3. Hyunjeong Lee, David Parsons, Gyuhyun Kwon, Jieun Kim, Krassie Petrova, Eunju Jeong, and Hokyoung Ryu. 2016. Cooperation begins: Encouraging critical thinking skills through cooperative reciprocity using a mobile learning game. Computers \& Education 97, 97-115. DOI:http://doi.org/10.1016/j.compedu.2016.03.006

4. Enrico Gandolfi. 2018. You have got a (different) friend in me: Asymmetrical roles in gaming as potential ambassadors of computational and cooperative thinking. E-Learning and Digital Media, 15(3), 128-145. DOI:doi.org/10.1177/2042753018757757

5. Ansgar E. Depping and Regan L. Mandryk. 2017. Cooperation and Interdependence: How Multiplayer Games Increase Social Closeness. 449-461. DOI: doi.org/10.1145/3116595.3116639.

6. Stefan Liszio and Maic Masuch. 2016. Designing Shared Virtual Reality Gaming Experiences in Local Multi-platform Games. DOI: doi.org/10.1007/978-3-319-46100723

7. Dave Mervik. 2017.Why, and how, Tarsier is bringing multiplayer into its upcoming PS VR puzzle mystery Statik. Retrieved from https://blog.eu.playstation.com/

8. Tracy Fullerton. 2008. Game Design Workshop: a Playcentric Approach to Creating Innovative Games (2nd. ed). CRC Press.

9. Tobias Greitemeyer, Eva Traut-Mattausch, and Silvia Osswald. 2012. How to ameliorate negative effects of violent video games on cooperation: Play it cooperatively in a team, Computers in Human Behavior 28, 4. 1465-1470, DOI:

https://doi.org/10.1016/j.chb.2012.03.009.

10. Tobias Greitemeyer and Christopher Cox. 2013. There's no "I" in team: Effects of cooperative video games on cooperative behavior. European Journal of Social Psychology 43, 4. 224-228. DOI: https://doi.org/10.1002/ejsp.1940

11. Mordecai Meirowitz. 1970. Mastermind. Game[board].

12. Chun-Wang Wei., Hao-Yun Kao, Hsin-Hsien Lu, and Yi Chun Liu. 2018. The effects of competitive gaming scenarios and personalized assistance strategies on english vocabulary learning. Journal of Educational Technology \& Society 21, 3. 146-158. 
13. Michael Mace, Nawal Kinany, Paul Rinne, Anthony Rayner, Paul Bentley, Etienne Burdet. 2017. Balancing the playing field: Collaborative gaming for physical training. Journal of Neuroengineering and Rehabilitation 14, 1. 116-18. DOI: doi.org/10.1186/s12984-017-0319-x.

14. Kylie Peppler, Joshua A. Danish, David Phelps. 2013. Collaborative Gaming: Teaching Children About Complex Systems and Collective Behavior. Simulation \& Gaming 44, 5. 683-705. DOI: https://doi.org/10.1177/1046878113501462

15. Nintendo. 2012. New Super Mario Bros. U. Game[WiiU].

16. John Harris, Mark Hancock, and Stacey Scott. 2016. Leveraging Asymmetries in Multiplayer Games: Investigating Design Elements of Interdependent Play. CHI PLAY '16 Proceedings of the 2016 Annual Symposium on Computer Human Interaction in Play. 350-361. 10.1145/2967934.2968113.

17. Cuihua Shen and Dmitri Williams. 2011. Unpacking time online: Connecting internet and massively multiplayer online game use with psychosocial well-being. Communication Research 38, 1. 123-149.

18. Extra Credits. 2015. Asymmetric Play - Can One Game Cater to Many Playstyles? (18 March 2015). Video. Retrieved July 1, 2019 from https://youtu.be/SQhxtfKH1f8

19. Conifer Research. 2002. Experience Design - How to Find Buried Treasure Using Experience Map. Retrieved July 1, 2019 from https://msu.edu/ jmonberg/491/User_files/ConiferExperienceMaps.pdf

20. Jesse Schell. 2018. The Future of Storytelling: How Medium Shapes Story. Video. Retrieved July 1, 2019 from https://www.youtube.com/watch?v=BjrO-di22v8

21. Amir Bozorgzadeh. 2018. VR arcade are playing a leading role in the consumer market. Retrieved from https://venturebeat.com

22. Peter Rubin. 2019. Review: Oculus Quest. Retrieved from https://www.wired.com/

23. Christo Petrov. 2019. 35 Virtual Reality Statistics That Will Rock the Market in 2019. Retrieved from https://techjury.net/

24. Steel Crate Games. 2015. Keep Talking and Nobody Explodes. Game [GearVR].

25. Tarsier Studios. 2017. Statik. Game[PSVR].

26. Ivanovitch Games. 2016. Final Soccer VR. Game[Vive/Oculus].

27. Free Lives. 2017. GORN. Game[Vive]. 
28. Raz Schwartz and William Steptoe. "The Immersive VR Self: Performance, Embodiment and Presence in Immersive Virtual Reality Environments." A Networked Self and Human Augmentics, Artificial Intelligence, Sentience, 2018. 108-116.

DOI:doi.org/10.4324/9781315202082-9.

29. John Koetsier. 2018. VR Needs more Social: 77\% of Virtual Reality Users Want More Social Engagement. Retrieved from

30. Shanhong Liu. 2019. Forecast for the number of active virtual reality users worldwide from 2014 to 2018 (in millions). Retrieved from https://www.statista.com/ 